\title{
Determination of PMMA Residues on a Chemical-Vapor- Deposited Monolayer of Graphene by Neutron Reflection and Atomic Force Microscopy
}

DOI:

10.1021/acs.langmuir.7b03117

\section{Document Version}

Accepted author manuscript

Link to publication record in Manchester Research Explorer

Citation for published version (APA):

Li, R., Li, Z., Pambou, E., Gutfreund, P., Waigh, T., Webster, J. R. P., \& Lu, J. (2018). Determination of PMMA Residues on a Chemical-Vapor-Deposited Monolayer of Graphene by Neutron Reflection and Atomic Force Microscopy. Langmuir, 34(5), 1827-1833. https://doi.org/10.1021/acs.langmuir.7b03117

\section{Published in:}

Langmuir

\section{Citing this paper}

Please note that where the full-text provided on Manchester Research Explorer is the Author Accepted Manuscript or Proof version this may differ from the final Published version. If citing, it is advised that you check and use the publisher's definitive version.

\section{General rights}

Copyright and moral rights for the publications made accessible in the Research Explorer are retained by the authors and/or other copyright owners and it is a condition of accessing publications that users recognise and abide by the legal requirements associated with these rights.

\section{Takedown policy}

If you believe that this document breaches copyright please refer to the University of Manchester's Takedown Procedures [http://man.ac.uk/04Y6Bo] or contact uml.scholarlycommunications@manchester.ac.uk providing relevant details, so we can investigate your claim.

\section{OPEN ACCESS}




\section{ANGMUIR}

Subscriber access provided by The University of Manchester Library

\section{Article}

Determination of PMMA Based Residues from a Chemical Vapor Deposited Monolayer of Graphene by Neutron Reflection and Atomic Force Microscopy

Ruiheng Li, Zongyi Li, Elias Pambou, Philipp Gutfreund, Thomas

Andrew Waigh, John Robert Peter Webster, and Jian Ren Lu

Langmuir, Just Accepted Manuscript • DOI: 10.1021/acs.langmuir.7b03117 • Publication Date (Web): 05 Jan 2018

Downloaded from http://pubs.acs.org on January 11, 2018

\section{Just Accepted}

"Just Accepted" manuscripts have been peer-reviewed and accepted for publication. They are posted online prior to technical editing, formatting for publication and author proofing. The American Chemical Society provides "Just Accepted" as a free service to the research community to expedite the dissemination of scientific material as soon as possible after acceptance. "Just Accepted" manuscripts appear in full in PDF format accompanied by an HTML abstract. "Just Accepted" manuscripts have been fully peer reviewed, but should not be considered the official version of record. They are accessible to all readers and citable by the Digital Object Identifier (DOI®). "Just Accepted" is an optional service offered to authors. Therefore, the "Just Accepted" Web site may not include all articles that will be published in the journal. After a manuscript is technically edited and formatted, it will be removed from the "Just Accepted" Web site and published as an ASAP article. Note that technical editing may introduce minor changes to the manuscript text and/or graphics which could affect content, and all legal disclaimers and ethical guidelines that apply to the journal pertain. ACS cannot be held responsible for errors or consequences arising from the use of information contained in these "Just Accepted" manuscripts. 


\title{
Determination of PMMA Residues on a Chemical Vapor Deposited Monolayer of
} Graphene by Neutron Reflection and Atomic Force Microscopy

\author{
Ruiheng $\mathrm{Li}^{a}$, Zongyi $\mathrm{Li}^{a}$, Elias Pambou ${ }^{a}$, Philipp Gutfreund ${ }^{b}$, Thomas A. Waigh ${ }^{a}$, John R.P. Webster ${ }^{c}$, Jian R. \\ $\mathrm{Lu}^{a *}$ \\ * E-mail: j.lu@manchester.ac.uk. Tel: (+44)-161-200-3926. \\ a Biological Physics Group, School of Physics and Astronomy, University of Manchester, Oxford Road, \\ Manchester, M13 9PL, UK. \\ ${ }^{\mathrm{b}}$ Institut Laue-Langevin, 71 avenue des Martyrs, 38000 Grenoble, France. \\ ${ }^{\mathrm{c}}$ STFC ISIS Facility, Rutherford Appleton Laboratory, Didcot OX11 0QX, U.K.
}

Keywords: Graphene film, CVD, film structure, neutron reflection, surface characterization, PMMA film.

\begin{abstract}
Chemical vapor deposition (CVD) is now a well-established method to create monolayer graphene films. In this method, poly(methyl methacrylate) (PMMA) films are often coated onto the monolayer graphene films to make them mechanically robust enough for transfer and further handling. However, it is found that the PMMA is hard to be removed entirely, and any residual polymers remaining can affect graphene's properties. We here demonstrate a method to determine the amount of the PMMA remaining on the graphene sheet fabricated from CVD by a combined study of Raman scattering, atomic force microscopy and neutron reflection. Neutron reflectivity is a powerful technique which is particularly sensitive to any interfacial structure, so it is able to investigate the density profile of the residual PMMA in the direction perpendicular to the graphene film surface. After the standard process of PMMA removal by acetone-IPA cleaning, we found the remaining PMMA film could be represented as a two layer model: an inner layer with thickness of $17 \AA$ and roughness of $3 \AA$ mixed with graphene and an outer diffuse layer with an average thickness of $31 \AA$ and a roughness of $4 \AA$ well mixed with water. Based on this model analysis, it was demonstrated that the remaining PMMA still occupied a significant fraction of the graphene film surface.
\end{abstract}




\section{Introduction}

Graphene, a two-dimensional sheet of carbon atoms arranged in a honeycomb network, was thought to be thermodynamically unstable until Geim and Novoselov isolated single-layer carbon sheets from highly oriented pyrolytic graphite (HOPG) at room temperature in 2004. ${ }^{1}$ Its unique two dimensional structure gives it a number of valuable properties, including an ultrahigh intrinsic electron mobility, ${ }^{2}$ a large specific surface area, ${ }^{3}$ good optical transmittance ${ }^{4}$ and excellent mechanical properties. ${ }^{5}$

Over the last decade, research into graphene and its properties has expanded significantly, driven by its potential uses in electronics, nanotechnology, healthcare and many other sectors. Large graphene films can be made into transparent electrodes with very low single sheet surface resistance $(125 \Omega / \square)$ which can be used for flexible touch displays. ${ }^{6}$ Other potential applications such as biosensors $^{7}$ and pollution control monitors ${ }^{8}$ have also been widely studied. Advancement of this technology is however still held back by the need to carry out a large-scale production of a uniform and reproducible sheet of graphene with minimal defects. In the technological and nanotechnology sectors, a uniform graphene film of reproducible thickness is necessary to achieve high quality and consistent performance. This is required for graphene to meet its potential and become a viable material on the massive scales, in the same way silicon came to a prominence in the 20th century.

Since the very first graphene sheets that were created by Geim and Novoselov using mechanical exfoliation, many alternative methods for the production of monolayer graphenes have been developed. ${ }^{9}$ Among the most commonly used for monolayer graphene layer production is chemical vapor deposition (CVD) ${ }^{10}$, in which method the monolayer graphene sheets can be provided at a relatively low cost so that they can now be readily obtained commercially, enabling widespread research into them. In the CVD production process, graphene is first grown on to a copper foil and then transferred to a target substrate. A polymer layer covered on the graphene film provides protection for the otherwise fragile and unstable $2 \mathrm{D}$ material so that it can be transferred and further handled. Poly(methyl methacrylate) (PMMA) is most commonly used as this polymeric protecting layer.

It is reported that the protective PMMA polymer is hard to be entirely removed from the surface of CVD graphene sheets by the standard cleaning procedure, including chemical treatment such as actone, isopropyl alcohol (IPA), ethanol, acidic acid, chlorinated solvents and their mixtures ${ }^{11,12}$. Thermal annealing has been carried out by a number of groups, but it is also difficult to remove unwanted contaminants from this approach. Furthermore, an oxidative gas could also be used for the clean process by breaking carbon double bonds ( $\mathrm{sp}^{2}$ bonds), ${ }^{13}$ but there is a risk that the gas may also damage the graphene arising from oxidation.

These PMMA residues will cause variations in the graphene monolayer's electronic properties across the sheet, due to nonuniformity existing across the surface. Residual PMMA will also reduce the electron mobility of graphene monolayers dramatically and hence mask the distinctive properties of this $2 \mathrm{D}$ material. ${ }^{13}$ Furthermore, the PMMA protective film could also lead to a nonuniform resistance across the monolayer surface, which in turn could bring about electronic failure or provide a 'burn-out' risk to electrical devices if not properly accounted for. However, many studies have only investigated CVD produced graphene films, but not addressed the presence of PMMA upon the surface, despite its influence upon the film properties.
Given the difficulty in eradicating the polymeric residues, it may alternatively be better to keep them but ensure that the PMMA film can be produced with controlled thickness and good uniformity to counterbalance possible defects or levels of unevenness so that reliable and consistent electronic properties for various applications can be provided. Therefore, the investigation of the intrinsic PMMA layer and its influence upon the electrical properties of a deposited graphene monolayer highlights the importance of further research into the structure and composition of deposited CVD graphene films. Raman spectroscopy is a comman technique for CVD graphene sample characterization. The $\mathrm{sp}^{3}$ hybridisation of the carbon atoms in the PMMA residues gives several peaks around $800 \mathrm{~cm}^{-1}\left(\mathrm{CH}_{3}\right.$ rocking), $1000 \mathrm{~cm}^{-1}$ (O-CH $\mathrm{CH}_{3}$ stretching), $1460 \mathrm{~cm}^{-1}\left(\mathrm{CH}_{3}\right.$ bending) and $1650 \mathrm{~cm}^{-1}$ $(\mathrm{O}=\mathrm{C}$ stretching) in Raman spectra. However, these peaks are usually too weak to be detected when the amount of PMMA adsorbed on the surface is low, typically in the thickness range of $\mathrm{nm}$. It is thus difficult to detect the existence of the residual material. Suk et al. have reported that the XPS spectra together with the Raman shifts of the $G$ peak position from graphene fieldeffect transistors (GFET) could help explain how the thin PMMA layer left on graphene causes p-type doping and lowers charge carrier mobility ${ }^{13}$. However, there still isn't a straightforward way to determine the distribution and amount of the residual PMMA. Neutron reflection (NR) is a powerful technique to reveal the thickness and composition of the multiple-layer system on the interface. By controlling the isotopic contrasts in parallel experiments, the amount of each component can be quantitatively analyzed with high depth resolution on the sub-nano level. However, NR had not been previously used to characterize graphene coated surfaces.

In this work, we have used neutron reflection to investigate the structure of a CVD monolayer graphene deposited on the silicon surface. The variation of the associated PMMA layer before and after acetone treatment was observed by its amount and volume fraction. Atomic force microscopy (AFM) images were taken to explore the morphological features of the sample surface. The monolayer graphene film was also characterized by Raman spectroscopy together with sheet resistance measurements.

\section{NR Technique and Analysis}

\subsection{Theory}

NR is a technique used for measuring the structure of thin films. In specular reflection, a highly collimated beam of neutrons is elastically scattered off an optically flat surface and reflected. The reflected intensity is measured as a function of wave vector or momentum transfer, $Q$,

$$
Q=\frac{4 \pi \sin \theta}{\lambda}
$$

where $\lambda$ is the incident neutron wavelength and $\theta$ is the incident angle. Analogous to the reflection of light, the neutron refractive index $(n)$ of non-absorbent materials can be defined by

$$
n=1-\frac{\lambda^{2} \rho}{2 \pi}
$$

where $\rho$ is the scattering length density $(S L D)$ of the material, which is defined by 


$$
\rho=\frac{\sum b_{c o h}}{v_{M}}
$$

Here $v_{M}$ is the material volume, and $\Sigma b_{c o h}$ is the sum of the coherent scattering length of each element that makes up the material. The total SLD of a molecule, film, or individual layer, is an additive quantity dependent on the coherent scattering length and the volume fraction of each isotopic component and thus its value could be determined for any material. The $S L D$ values that will be used in this study are listed in Table 1.

The $S L D$ of a material can be determined if its molecular composition is known. While the $S L D$ of PMMA was calculated simply from its density and chemical formula $\left(\left(\mathrm{C}_{5} \mathrm{O}_{2} \mathrm{H}_{8}\right) n\right)$, determination of the $S L D$ of graphene is less straightforward. In order to mathematically determine the $S L D$ of graphene, the volume occupied by a graphene atom is required. Graphene is a relatively new material with contradictory reports in the literature over its structural properties and thus its exact density is disputed. The carbon-carbon bond length is known to be $1.42 \AA$ (an average length of $\mathrm{sp}^{2}$ bond and $\mathrm{sp}$ bond), which gives the hexagonal unit cell an area of $5.24 \AA^{2}$ and that occupied by a graphene atom to be $2.62 \AA^{2}$ ( 2 atoms per unit cell). The height of a perfectly formed graphene monolayer film is reported to be $3.45 \AA$ (the distance between neighboring layers in highly ordered pyrolytic graphene), to give the total volume occupied by a carbon atom in graphene to be $9.04 \AA^{3}$. The scattering length of carbon, $b_{\text {carbon }}$ is $6.65 \times 10^{-5}$ $\AA^{-2}$, which gives a total graphene $S L D$ of $7.35 \times 10^{-6} \AA^{-2}$.

\begin{tabular}{cc}
\hline Material & $\rho\left(\AA^{-2}\right)\left(\times 10^{-6}\right)$ \\
\hline $\mathrm{H}_{2} \mathrm{O}$ & -0.56 \\
$\mathrm{D}_{2} \mathrm{O}$ & 6.35 \\
Silicon $(\mathrm{Si})$ & 2.07 \\
Silicon Oxide $\left(\mathrm{SiO}_{2}\right)$ & 3.49 \\
Air & 0 \\
Graphene & 7.35 \\
PMMA & 0.79
\end{tabular}

Table 1. Scattering length density values of the materials used in this work for neutron reflectivity studies.

By taking advantage of variations in scattering lengths between elemental isotopes, a technique referred to as isotopic substitution can be exploited to alter the $S L D$ of a compound without significantly altering its chemical structure. Therefore, a particular region of interest can be 'labelled' to highlight a specific feature. $^{14,15}$ For solid/liquid interfaces, isotopic substitution is commonly used to alter the $S L D$ of the solvent used. An example of this is the substitution of $\mathrm{H}_{2} \mathrm{O}$ with $\mathrm{D}_{2} \mathrm{O}$. Combinations of the two forms of water can be used to create solutions of well-defined $S L D$ s. Water contrast matched to air (CMAir) is commonly used in neutron reflection and is made up of $8.1 \% \mathrm{D}_{2} \mathrm{O}$ and $91.9 \% \mathrm{H}_{2} \mathrm{O}$, giving an overall $S L D$ of zero.

\subsection{Neutron Reflection Data Collection}

Specular NR measurements were carried out using the time-offlight reflectometer D17 at the ILL, Grenoble, France. ${ }^{16}$ To create an environment for measurement, samples were assembled in a solid/liquid configuration and were measured using purpose built liquid cells made of Perspex and clamped to the silicon ( $\mathrm{Si}$ ) block by means of a steel assembly. On the vertical reflectometer of D17, measurements were carried out on a variable angle sample stage at incidence angles of $0.8^{\circ}$ and $3.2^{\circ}$, with a $Q$-range of 0.008 to $0.16 \AA^{-1}$. Air/Si interface measurements were also carried out with the Si block placed flat upon the sample stage and fixed in place using double-sided tape. Si/air measurements allow for a stronger NR signal and give a critical edge of total reflection due to the respective $S L D \mathrm{~s}$ of the two bulk phases (analogous to Snell's law in optics). The solid/liquid and solid/air measurements analyzed in this paper were all conducted at ambient temperature $(\sim 298 \mathrm{~K})$.

\subsection{NR Data Analysis - Volume Fraction Approach}

The neutron reflectivity profiles were analyzed using Motofit ${ }^{17}$ (based on Igor Pro 6.22 ) which uses an optical matrix formalism based on the Abele's layer method to compare and simultaneously fit experimental data obtained under multiple solvent contrasts to a single reflectivity model. ${ }^{18}$ The reflectivity model contains a series of stacked thin layers and each layer was described by three parameters, scattering length density (SLD), thickness $(\tau)$ and roughness $(\delta)$. Considering a layer consisted of $\mathrm{n}$ materials with SLD of $\rho_{n}$ and volume fraction of $V_{n}$, the overall layer SLD is given by:

$$
\rho_{\text {layer }}=\sum_{i=1}^{n} V_{n} \rho_{n}
$$

The roughness is implemented in terms of an error function according to Nevot and Croces. ${ }^{19}$

Least-squares minimization (Nelder-Mead Simplex) was used to determine the best-fit parameters by comparing the reflectivity simulated based on the model to the experimental data. In all cases, the simplest possible model which satisfactorily described the data under different isotopic contrasts was selected.

\section{Sample Preparation and Characterization}

\subsection{Chemical Vapour Deposition (CVD)}

Large area monolayer graphene was grown on a copper foil with the CVD method. ${ }^{20,21}$ PMMA $\left(\mathrm{M}_{\mathrm{w}}=950 \mathrm{k}\right.$, PDI $\left.=2.1\right)$ was deposited by spin coating on the copper-foil-grow graphene. The PMMA layer can be used as a carrier for transferring the monolayer graphene to $\mathrm{SiO}_{2} / \mathrm{Si}$ substrate. ${ }^{22}$ In the transformation process, the PMMA/graphene/copper foil was first floated on the surface of $0.04 \mathrm{~g} / \mathrm{ml}$ ammonium persulfate (APS) solution (the copper side faced down $)^{23}$ for several hours until the copper was totally etched. After that, the APS solution was replaced by UHQ water (deionised ultrahigh quality water) and the graphene/PMMA sheet was left floating on the water surface. The target $\mathrm{SiO}_{2} / \mathrm{Si}$ substrate was then immersed in the the water underneath the PMMA/graphene sheet, and the substrate was lifted so that the PMMA/graphene sheet could attach to the substrate. The graphene monolayer adhered tightly to the $\mathrm{SiO}_{2} / \mathrm{Si}$ substrate after subsequent baking on a hot plate.

\subsection{PMMA Removal}

In this study, acetone and isopropyl alcohol were used to perform a standard cleaning prodedure. each sample was first dipped into acetone for $10 \mathrm{~min}$, then in isopropyl alcohol (IPA) for $10 \mathrm{~min}$, followed by UHQ (ultrapure water) rinsing for further $10 \mathrm{~min}^{23-}$ ${ }^{26}$ This cleaning protocol helped to reduce the amount of adsorbed 
PMMA. Nitrogen gas was used to dry the samples after each process of PMMA cleaning.

\subsection{Raman Imaging}

Raman spectroscopy was used to evaluate the quality of the monolayer graphene deposited on the silicon block $\left(\mathrm{SiO}_{2} / \mathrm{Si}\right)$ for NR measurements. The main Raman peaks of graphene include the $\mathrm{G}$ peak $\left(\sim 1560 \mathrm{~cm}^{-1}\right)$, the 2D peak $\left(2700 \mathrm{~cm}^{-1}\right)$ and sometimes the D peak $\left(\sim 1360 \mathrm{~cm}^{-1}\right)$ arising from the defects of the sample. ${ }^{27}$ The typical range of this spectrum is measured over 1250-3250 $\mathrm{cm}^{-1}$, covering all the characteristic peaks of the graphene film. Figure 1 shows a typical set of Raman measurements taken on 7 different positions of the block's surface and the spectral consistency indicated that the supported graphene layer was homogeneous across the sample area. The G and 2D peaks are positioned around $1595 \mathrm{~cm}^{-1}$ and $2695 \mathrm{~cm}^{-1}$ respectively. Evidence for monolayer graphene is that the $2 \mathrm{D}$ peak is very symmetrical and has a narrow full width at half maximum $\left(60 \mathrm{~cm}^{-}\right.$ $\left.{ }^{1}\right)$. A small D peak around $1350 \mathrm{~cm}^{-1}$ is also visible, indicating some defects. This peak is associated with the existence of PMMA. $^{28}$ It is noticeable that the background noise increased around the D peak which is from the amorphous carbons on the surface. $^{29}$

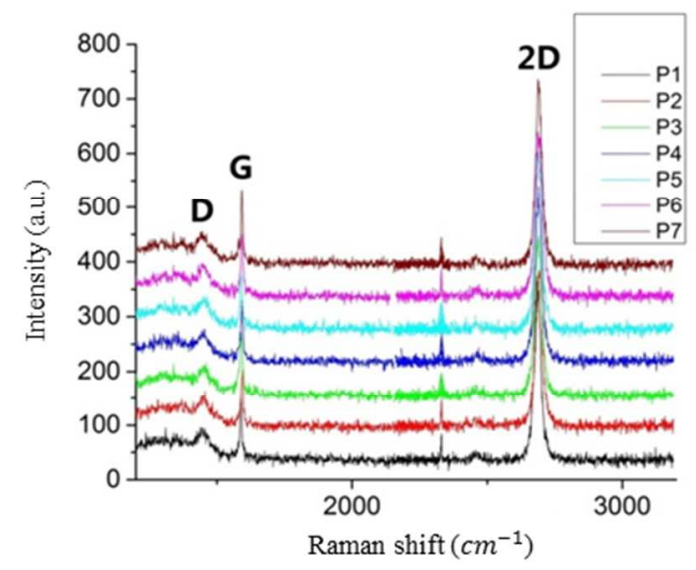

Figure 1. Raman spectra for CVD monolayer graphene on a silicon block. The scattered intensity is shown as a function of the Raman shift (the wave number). The Raman spectra were taken at 7 different points and were labelled as $P 1 \sim P 7$ on a typical graphene monolayer of $2 \times 3 \mathrm{~cm}^{2}$.

Against the general criteria, as outlined above, the Raman spectra shown in Figure 1 are virtually identical, showing that the monolayer graphene deposited on the silicon block $\left(\mathrm{SiO}_{2} / \mathrm{Si}\right)$ was uniform throughout the film surface.

\subsection{Atomic Force Microscopy (AFM)}

AFM measurements of the CVD graphene topography and structure were obtained using a Bruker-Dimension 3100 scanning probe microscope at Rutherford Appleton Laboratory (RAL), Harwell Campus. Scans were carried out in 'tapping mode' using a gold plated $\mathrm{Si}$ cantilever of tip height of 10-15 $\mu \mathrm{m}$ and tip curvature of radius $10 \mathrm{~nm}$. (NT-MDT, NSG10). Graphene samples for AFM were prepared in the same way as for NR.

\subsection{Sheet Electrical Resistance}

The electrical resistance of the CVD graphene sheet was measured with a 4-point probe (Jandel model RM3000). The intrinsic sheet resistance from a graphene monolayer was reported to be about $40 \Omega / \square .{ }^{28}$ Even though the sample has an average sheet resistance of few hundreds $\Omega / \square$, it is still considered acceptable for a CVD sample. The high resistance is indicative of the presence of PMMA as well as the defects in the graphene monolayer.

\section{Results and Discussion}

\subsection{Imaging Analysis}

AFM imaging scans were obtained from CVD graphene sample after acetone-IPA cleaning process which reduced the PMMA polymer. AFM scans were performed over a $10 \mu \mathrm{m}^{2}$ region at different locations on the silicon substrate, with a representative one shown in Figure 2.

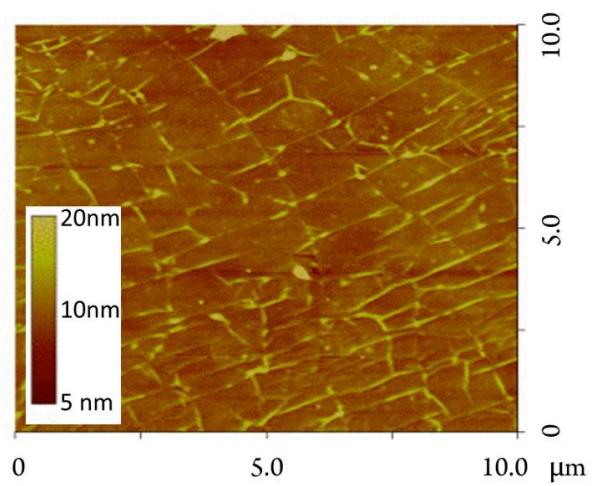

Figure 2. The AFM image shows the surface of a CVD monolayer of graphene on a silicon surface after the PMMA removal treatment, revealing the existence of PMMA residues as evident from the web pattern on the wrinkled graphene. ${ }^{30}$ The inset shows the calibration of heights above the surface.

The AFM image of CVD graphene with PMMA coating had been reported to have a web-like pattern with a height up to tens nanometers. ${ }^{31}$ The pattern is presented because of the absorbed PMMA on the wrinkles of graphene layers. After acetone-IPA cleaning was performed, our AFM image in Figure 2 shows the web-like pattern still remains on the graphene surface. However, AFM can only inform us about the outer surface of the film and cannot reveal details regarding the inner sturcture of graphene and PMMA complex.

\subsection{Sheet Resistance}

The sheet resistance values of the transferred graphene monolayer were measured in different positions and the values varied between 750 and $1050 \Omega / \square$, with no apparent difference before and after acetone washing within the variation range. Figure 3 compares the average value obtained from this work measured from the sheet surface before acetone-IPA washing (point 1) with the theoretical value corresponding to the pure graphene and the different values reported by Park et $\mathrm{al}^{32-35}$ (points 2-4, denote values obtained for acetone washing, acetone washing and heat treatment and heat treatment only, respectively). 


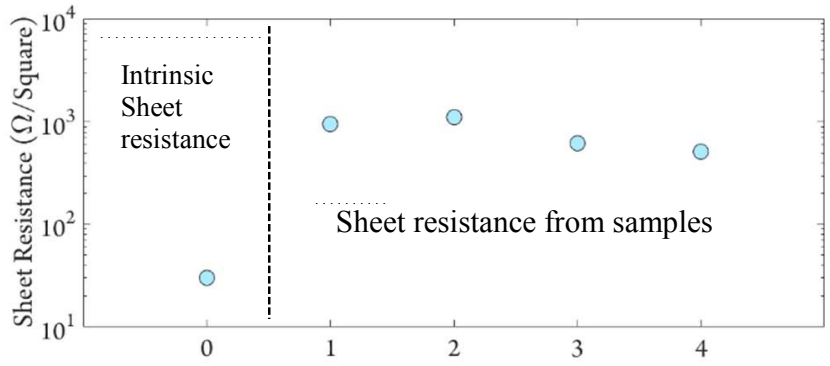

Figure 3. Sheet resistances measured from a graphene sample using the 4-point probe method. Position 0 is the theoretical value of sheet resistance for a graphene monolayer. ${ }^{36}$ Position 1 is the sheet resistance of the sample used in this paper before PMMA removal, while 2-4 are data from previous work ${ }^{37}$ in which the PMMA layer was removed by acetone treatment, combined acetone and heat treatments $\left(a t 400^{\circ} \mathrm{C}\right)$ and heat treatment (at $400^{\circ} \mathrm{C}$ ), respectively.

As shown in Figure 3, the intrinsic sheet resistance of a pure graphene monolayer is about $40 \Omega / \square$ while the actual samples give sheet resistance values $5-20$ times the theoretical value. The defects from the graphene layer itself and the presence of the residual PMMA layer can alter the sheet resistance readings, but their exact influences remain to be characterized. Solvent washing in our case did not alter resistance readings significantly.

\subsection{Neutron Reflection (NR)}

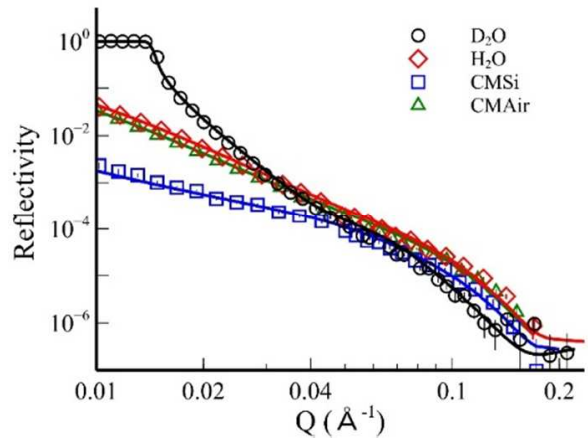

Figure 4. Neutron reflectivity profiles as a function of momentum transfer (Q) measured from four different contrasts: $\mathrm{D}_{2} \mathrm{O}, \mathrm{H}_{2} \mathrm{O}$, CMAir (contrast matched to air) and CMSi (contrast matched to silicon), with the continuous lines denoting the best model fits.

Figure 4 shows a set of neutron reflectivity curves plotted as a function of momentum transfer $(Q)$ measured from four different contrasts: $\mathrm{D}_{2} \mathrm{O}, \mathrm{H}_{2} \mathrm{O}$, CMAir (contrast matched to air) and CMSi (contrast matched to silicon) before acetone-IPA washing. Structural information about thickness and composition is usually obtaned by layer modelling, with the simplest one being the single layer. It is definded by the parameters of layer SLD $\rho$, thickness $\tau$, and roughness $\delta$. By searching for the simplest model that could fit the data, the number of parameters used in defining the model would be kept at the smallest, thus ensuring better physcial validity. Where possible, $\delta$ was also kept at the minimum in the fitting. The solid lines as shown in Fugure 4 represent the best simultaneous fits to the data measured under the multiple contrasts. This model consists of an inner uniform graphene/PMMA mixture layer (G/P layer) and an outer diffuse PMMA polymer layer (P-layer) into the aqueous phase. The key parameters of the best-fit model are listed in Table 2.

\begin{tabular}{|c|c|c|c|c|}
\hline Layer & $\begin{array}{l}\text { Thickness } \\
\text { (Å) }\end{array}$ & $\begin{array}{c}\text { Roughness } \\
\text { (Å) }\end{array}$ & Contrast & $\begin{array}{c}\text { SLD } \\
\left(\AA^{-2} \times 10^{-6}\right)\end{array}$ \\
\hline \multirow{4}{*}{$\begin{array}{l}\text { Inner } \\
\text { Layer }\end{array}$} & \multirow{4}{*}{17} & \multirow{4}{*}{3} & $\mathrm{D}_{2} \mathrm{O}$ & 5.12 \\
\hline & & & CMSi & 3.08 \\
\hline & & & CMAir & 2.05 \\
\hline & & & $\mathrm{H}_{2} \mathrm{O}$ & 1.77 \\
\hline \multirow{4}{*}{$\begin{array}{l}\text { Outer } \\
\text { Layer }\end{array}$} & \multirow{4}{*}{50} & \multirow{4}{*}{24} & $\mathrm{D}_{2} \mathrm{O}$ & 3.47 \\
\hline & & & CMSi & 1.43 \\
\hline & & & CMAir & 0.40 \\
\hline & & & $\mathrm{H}_{2} \mathrm{O}$ & 0.12 \\
\hline
\end{tabular}

Table 2. Key structural parameters obtained from the best 2-layer fit to the neutron reflectivity profiles measured from the CVD graphene sample under different solvent isotopic contrasts.

The simultaneous fits to the reflectivity profiles measured under different isotopic contrasts helped us to determine the layer composition under the 2-layer model. The mixture within the inner layer is comprised of PMMA, graphene and water, equation 4 can be rewritten with the volume fraction for each of them, $\mathrm{V}_{\text {graphene, }}, \mathrm{V}_{\mathrm{PMMA}}$, and $\mathrm{V}_{\text {water }}$ :

$\rho_{\text {layer }}=V_{\text {graphene }} \rho_{\text {graphene }}+V_{\text {PMMA }} \rho_{\text {PMMA }}+V_{\text {water }} \rho_{\text {water }}$

where $\rho_{\text {layer }}$ denotes the $S L D$ of the inner layer and was obtained from the best 2-layer model fitting. At the solid/water interface, the interfacial film was measured under 4 solvent contrasts, but as shown in Figure $4 \mathrm{H}_{2} \mathrm{O}$ and CMSAir are very close. Although the similarity adds confidence in the consistency of the data, they did not contribute to the resolution of layer composition. Thus, we can write the above equation under the 3 uncorrelated isotopic contrasts with the $\rho_{\text {layer }}$ value being determined from the respective best fit. Also, $\mathrm{V}_{\text {graphene }}+\mathrm{V}_{\text {PMMA }}+\mathrm{V}_{\text {water }}=1$. This means we still overdetermined the variables, but because the 3 contrasts have different sensitivity, the over-determination helped us to ensure the validity of the results. Figure 5 depicts how the volume fraction of each material is distributed against the vertical distance from the interface.

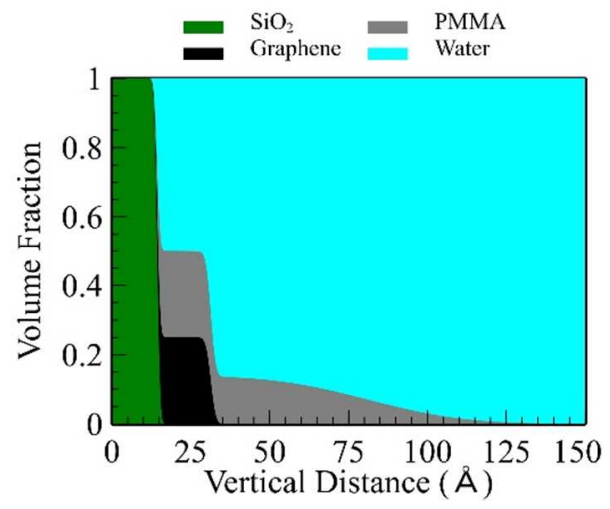

Figure 5. The volume fraction distribution as a function of distance perpendicular to the surface calculated from the neutron reflectivity data as shown in Figure 4, with the influence of roughness being implemented in terms of an error function according to Nevot and Croces. ${ }^{19}$

As shown in Figure 5, the inner layer has a thickness of $17 \AA$ and a roughness of $3 \AA$ on both top and bottom layer interface. Graphene is only present in the inner layer with a volume fraction of 0.25 . The volume fraction of PMMA remains constant at 0.25 
in the thin inner layer but then decays rapidly from the outer surface of the inner layer throughout the outer layer. The long decay tail results from the large roughness between the outer layer and the water bulk. The large roughness of $24 \AA$ implies a diffuse PMMA surface on the top of the CVD graphene sample. Under water this surface layer is heavily hydrated.

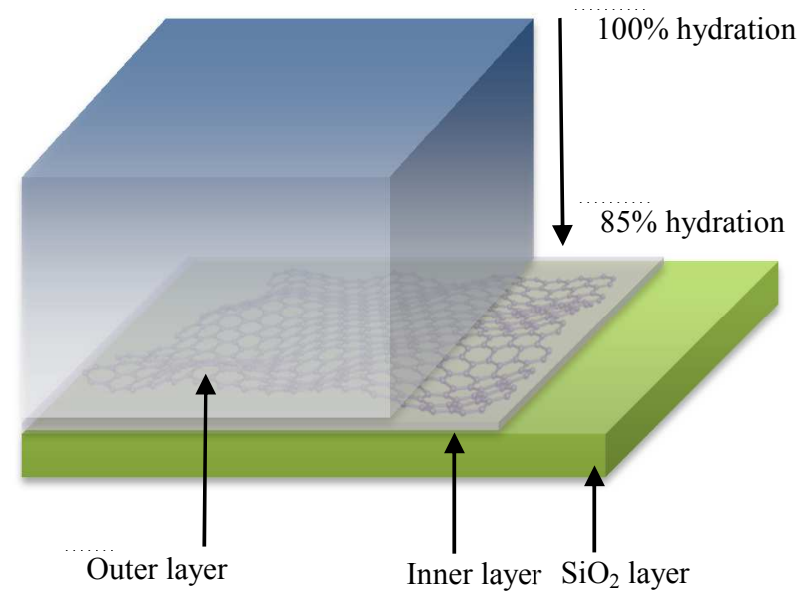

Figure 6. Schematic diagram of the model, with the color green denoting the silicon oxide and color grey representing PMMA.

Theoretically, a flat graphene monolayer can be described as a fully occupied layer with a thickness of $3.45 \AA$ and SLD of 7.35 $\times 10^{-6} \AA^{-2}$. However, our results reveal that the graphene distributed in a layer of $17 \AA$ and occupied 0.25 of the volume in this layer. It indicates a wavy graphene layer mixed with PMMA was on the silicon surface. The fluctuating structure of the graphene sheet leads to its low volume fraction and the large distributed range. Overall, this uneven structure of graphene layer together with the coated PMMA is illustrated in Figure 6.

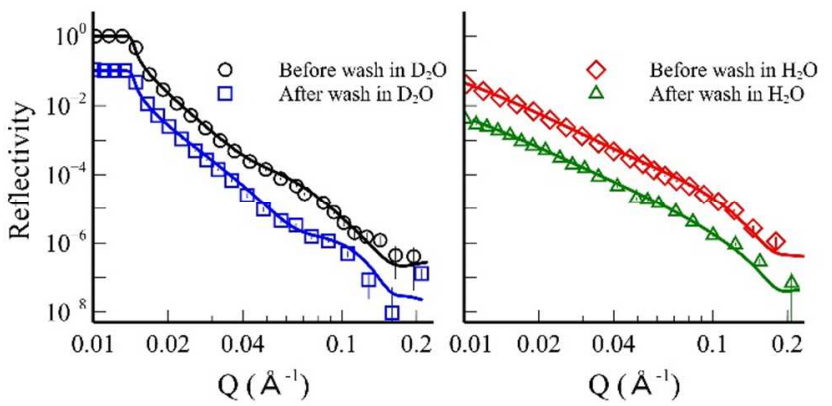

Figure 7. Neutron reflectivity measured as a function of wave vector transfer (Q) from the CVD graphene in $\mathrm{H}_{2} \mathrm{O}$ and $\mathrm{D}_{2} \mathrm{O}$. The measurements were taken before and after acetone treatment. For clarity, the profiles after wash were plotted with a 0.1 offset.

The neutron reflectivity profiles of the CVD graphene sample were also collected in $\mathrm{H}_{2} \mathrm{O}$ and $\mathrm{D}_{2} \mathrm{O}$ before and after acetone-IPA washing, and plotted in Figure 7. These two specific bulk water contrasts were selected to create the largest SLD differences against graphene and PMMA so that the structural changes of the two components were well highlighted.

In $\mathrm{H}_{2} \mathrm{O}$, graphene became highlighted but little change was detected from the inner graphene layer, as evident from the almost identical reflectivity profiles in Figure 7. In contrast, the change observed from the $\mathrm{D}_{2} \mathrm{O}$ profiles indicated PMMA removal. Data analysis revealed the reduction of PMMA from the outer diffuse
PMMA layer, which is represented by a thickness change from 50 $\AA$ to $31 \AA$ and a smaller roughness of $4 \AA$ instead of $24 \AA$. Thus, acetone-IPA rinsing helped reduce the amount of PMMA in the diffuse layer but did not remove all of it.

\section{Conclusions}

In this study, structure details of CVD graphene films deposited on silicon substrate were revealed by neutron reflection (NR). The interfacial film was described by a 2-layer model, with each layer being characterized by layer thickness, roughness and volume fraction of graphene and PMMA. The results revealed that the inner layer is comprised of a wavy graphene monolayer of $17 \AA$ together with PMMA while the outer layer is featured by a thick, highly difuse PMMA distribution only.

Furthermore, NR measurements were also used to characterize the same graphene film after a standard acetone-IPA cleaning. The results revealed that whilst little influence was made to the inner graphene/PMMA layer solvent washing did remove some of the PMMA present in the outer PMMA layer, making it thinner and smoother. However, the results indicated that the coatted PMMA film could not be entirely removed from the CVD graphene, consistent with the observation reported by other groups ${ }^{11-13}$.

NR is advantageous in the quantitive determination of residual PMMA in terms of amount and structure, making it attractive to characterise how residual PMMA affects the mechanical and electrical properties of the CVD graphene monolayers. It will thus help develop better defined CVD graphene films.

\section{Acknowledgements}

We thank ISIS Neutron Facility and Institut Laue Langevin for the support of the neutron reflectivity measurements, the University of Manchester for funding the research under the Graphene Grand Challenges scheme, and 2D-Tech Ltd for CVD production of the graphene films and the Raman measurements. This work also benefited from the grant support from EPSRC (EP/F062966/1) and Innovate UK (KTP008143, KTP009043).

\section{References}

(1) Novoselov, K. S. Electric Field Effect in Atomically Thin Carbon Films. Science (80-. ). 2004, 306 (5696), 666-669.

(2) Morozov, S. V.; Novoselov, K. S.; Katsnelson, M. I.; Schedin, F.; Elias, D. C.; Jaszczak, J. A.; Geim, A. K. Giant Intrinsic Carrier Mobilities in Graphene and Its Bilayer. Phys. Rev. Lett. 2008, $100(1), 16602$.

(3) Kuchta, B.; Firlej, L.; Mohammadhosseini, A.; Boulet, P.; Beckner, M.; Romanos, J.; Pfeifer, P. Hypothetical High-SurfaceArea Carbons with Exceptional Hydrogen Storage Capacities: Open Carbon Frameworks. J. Am. Chem. Soc. 2012, 134 (36), 1513015137.

(4) Mak, K. F.; Sfeir, M. Y.; Wu, Y.; Lui, C. H.; Misewich, J. A.; Heinz, T. F. Measurement of the Opptical Conductivity of Graphene. Phys. Rev. Lett. 2008, 101 (19), 196405.

(5) Frank, I. W.; Tanenbaum, D. M.; van der Zande, A. M.; McEuen, P. L. Mechanical Properties of Suspended Graphene Sheets. J. Vac. Sci. Technol. B Microelectron. Nanom. Struct. 2007, 25 (6), 2558.

(6) Bae, S.; Kim, H.; Lee, Y.; Xu, X.; Park, J. S.; Zheng, Y.; Balakrishnan, J.; Lei, T.; Ri Kim, H.; Song, Y. Il; et al. Roll-to-Roll Production of 30-Inch Graphene Films for Transparent Electrodes. Nat. Nanotechnol. 2010, 5 (8), 574-578. 
(7) Shao, Y.; Wang, J.; Wu, H.; Liu, J.; Aksay, I. A.; Lin, Y. Graphene Based Electrochemical Sensors and Biosensors: A Review. Electroanalysis 2010, 22 (10), 1027-1036.

(8) Singh, K.; Ohlan, A.; Pham, V. H.; R., B.; Varshney, S.; Jang, J.; Hur, S. H.; Choi, W. M.; Kumar, M.; Dhawan, S. K.; et al. Nanostructured graphene/Fe3O4 Incorporated Polyaniline as a High Performance Shield against Electromagnetic Pollution. Nanoscale 2013, 5 (6), 2411.

(9) Novoselov, K. S.; Fal'Ko, V. I.; Colombo, L.; Gellert, P. R.; Schwab, M. G.; Kim, K. A Roadmap for Graphene. Nature 2012, 490 (7419), 192-200.

(10) Reina, A.; Jia, X.; Ho, J.; Nezich, D.; Son, H.; Bulovic, V.; Dresselhaus, M. S.; Jing, K. Large Area, Few-Layer Graphene Films on Arbitrary Substrates by Chemical Vapor Deposition. Nano Lett. 2009, 9 (1), 30-35.

(11) Gong, C.; Floresca, H. C.; Hinojos, D.; McDonnell, S.; Qin, X.; Hao, Y.; Jandhyala, S.; Mordi, G.; Kim, J.; Colombo, L.; et al. Rapid Selective Etching of PMMA Residues from Transferred Graphene by Carbon Dioxide. J. Phys. Chem. C 2013, 117 (44), 23000-23008.

(12) Liang, X.; Sperling, B. A.; Calizo, I.; Cheng, G.; Hacker, C. A.; Zhang, Q.; Obeng, Y.; Yan, K.; Peng, H.; Li, Q.; et al. Toward Clean and Crackless Transfer of Graphene. ACS Nano 2011, 5 (11), 9144-9153.

(13) Suk, J. W.; Lee, W. H.; Lee, J.; Chou, H.; Piner, R. D.; Hao, Y.; Akinwande, D.; Ruoff, R. S. Enhancement of the Electrical Properties of Graphene Grown by Chemical Vapor Deposition via Controlling the Effects of Polymer Residue. Nano Lett. 2013, 13 (4), 1462-1467.

(14) Lu, J. R.; Li, Z. X.; Smallwood, J.; Thomas, R. K.; Penfold, J. Detailed Structure of the Hydrocarbon Chain in a Surfactant Monolayer at the Air/Water Interface: Neutron Reflection from Hexadecyltrimethylammonium Bromide. J. Phys. Chem. 1995, 99 (20), 8233-8243.

(15) Lu, J. R.; Simister, E. A.; Lee, E. M.; Thomas, R. K.; Rennie, A. R.; Penfold, J. Direct Determination by Neutron Reflection of the Penetration of Water into Surfactant Layers at the Air/Water Interface. Langmuir 1992, 8 (7), 1837-1844.

(16) Cubitt, R.; Fragnetto, G. D17: The New Reflectometer at the ILL. Appl. Phys. A Mater. Sci. Process. 2002, 74, S329-S331.

(17) Nelson, A. Co-Refinement of Multiple-Contrast neutron/XRay Reflectivity Data Using MOTOFIT. J. Appl. Crystallogr. 2006, 39 (2), 273-276.

(18) Fragneto-Cusani, G. Neutron Reflectivity at the Solid / Liquid Interface : Examples of Applications in Biophysics. J. Phys. Condens. Matter 2001, 13 (21), 4973.

(19) Névot, L.; Croce, P. Caractérisation des surfaces par réflexion rasante de rayons $\mathrm{X}$. Application à l'étude du polissage de quelques verres silicates. Rev. Phys. Appliquée 1980, 15 (3), 761-779.

(20) Li, X.; Cai, W.; An, J.; Kim, S.; Nah, J.; Yang, D.; Piner, R.; Velamakanni, A.; Jung, I.; Tutuc, E.; et al. Large-Area Synthesis of High-Quality and Uniform Graphene Films on Copper Foils. Science (80-. ). 2009, 324 (5932), 1312-1314.

(21) Li, X.; Magnuson, C. W.; Venugopal, A.; Tromp, R. M.; Hannon, J. B.; Vogel, E. M.; Colombo, L.; Ruoff, R. S. Large-Area Graphene Single Crystals Grown by Low-Pressure Chemical Vapor Deposition of Methane on Copper. J. Am. Chem. Soc. 2011, 133 (9), 2816-2819.

(22) Suk, J. W.; Kitt, A.; Magnuson, C. W.; Hao, Y.; Ahmed, S.; An, J.; Swan, A. K.; Goldberg, B. B.; Ruoff, R. S. Transfer of CVD-Grown Monolayer Graphene onto Arbitrary Substrates. ACS Nano 2011, 5 (9), 6916-6924.

(23) Wang, Y. Y.; Burke, P. J. A Large-Area and Contamination-Free Graphene Transistor for Liquid-Gated Sensing Applications. Appl. Phys. Lett. 2013, 103 (5).

(24) Wood, J. D.; Doidge, G. P.; Carrion, E. A.; Koepke, J. C.; Kaitz, J. A.; Datye, I.; Behnam, A.; Hewaparakrama, J.; Aruin, B.;
Chen, Y.; et al. Annealing Free, Clean Graphene Transfer Using Alternative Polymer Scaffolds. Nanotechnology 2015, 26 (5), 55302.

(25) Van Der Zande, A. M.; Barton, R. A.; Alden, J. S.; Ruiz-

Vargas, C. S.; Whitney, W. S.; Pham, P. H. Q.; Park, J.; Parpia, J. M.; Craighead, H. G.; McEuen, P. L. Large-Scale Arrays of Single-Layer Graphene Resonators. Nano Lett. 2010, 10 (12), 4869-4873.

(26) Regan, W.; Alem, N.; Alemán, B.; Geng, B.; Girit, Ç.; Maserati, L.; Wang, F.; Crommie, M.; Zettl, A.; Regan, W.; et al. A Direct Transfer of Layer-Area Graphene A Direct Transfer of LayerArea Graphene. Appl. Phys. Lett. 2015, 113102 (2010), 1-4.

(27) Malard, L. M.; Pimenta, M. A.; Dresselhaus, G.; Dresselhaus, M. S. Raman Spectroscopy in Graphene. Phys. Rep. 2009, 473 (5-6), 51-87.

(28) Lupina, G.; Kitzmann, J.; Lukosius, M. Deposition of Thin Silicon Layers on Transferred Large Area CVD Graphene. arXiv Prepr. arXiv ... 2014, 103 (26).

(29) Hong, J.; Park, M. K.; Lee, E. J.; Lee, D.; Hwang, D. S.; Ryu, S. Origin of New Broad Raman D and G Peaks in Annealed Graphene. Sci. Rep. 2013, 3, 2700.

(30) Pirkle, A.; Chan, J.; Venugopal, A.; Hinojos, D.; Magnuson, C. W.; McDonnell, S.; Colombo, L.; Vogel, E. M.; Ruoff, R. S.; Wallace, R. M. The Effect of Chemical Residues on the Physical and Electrical Properties of Chemical Vapor Deposited Graphene Transferred to SiO2. Appl. Phys. Lett. 2011, 99 (12), 1013.

(31) Van Ngoc, H.; Qian, Y.; Han, S. K.; Kang, D. J. PMMAEtching-Free Transfer of Wafer-Scale Chemical Vapor Deposition Two-Dimensional Atomic Crystal by a Water Soluble Polyvinyl Alcohol Polymer Method. Sci. Rep. 2016, 6, 33096.

(32) La Notte, L.; Villari, E.; Palma, A. L.; Sacchetti, A.; Michela Giangregorio, M.; Bruno, G.; Di Carlo, A.; Bianco, G. V.; Reale, A. Laser-Patterned Functionalized CVD-Graphene as Highly Transparent Conductive Electrodes for Polymer Solar Cells. Nanoscale 2017, 9 (1), 62-69.

(33) Wu, X.; Asselberghs, I.; Politou, M.; Contino, A.; Radu, I.; Huyghebaert, C.; Tokei, Z.; Soree, B.; De Gendt, S.; De Feyter, S.; et al. Doping of Graphene for the Application in Nano-Interconnect. Microelectron. Eng. 2017, 167, 42-46.

(34) Deepshikha. Effect of Doping on Photovoltaic Characteristics of Graphene. Russ. J. Phys. Chem. A 2016, 90 (13), 2609-2615.

(35) Sun, J.; Chen, Z.; Yuan, L.; Chen, Y.; Ning, J.; Liu, S.; Ma, D.; Song, X.; Priydarshi, M. K.; Bachmatiuk, A.; et al. Direct Chemical-Vapor-Deposition-Fabricated, Large-Scale Graphene Glass with High Carrier Mobility and Uniformity for Touch Panel Applications. ACS Nano 2016, 10 (12), 11136-11144.

(36) Chen, J. H.; Jang, C.; Xiao, S.; Ishigami, M.; Fuhrer, M. S. Intrinsic and Extrinsic Performance Limits of Graphene Devices on $\mathrm{SiO}$ 2. Nat. Nanotechnol. 2008, 3 (4), 206-209.

(37) Park, J. H.; Jung, W.; Cho, D.; Seo, J. T.; Moon, Y.; Woo, S. H.; Lee, C.; Park, C. Y.; Ahn, J. R. Simple, Green, and Clean Removal of a Poly(methyl Methacrylate) Film on Chemical Vapor Deposited Graphene. Appl. Phys. Lett. 2013, 103 (17).

ToC:

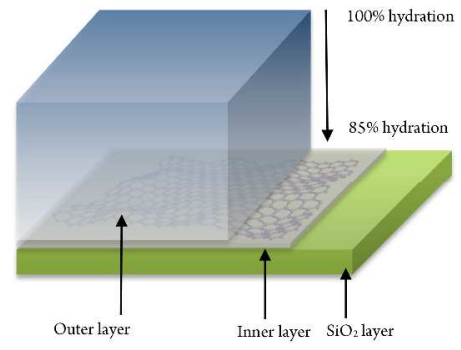

